University of Windsor

Scholarship at UWindsor

2001

\title{
Quantum phase retrieval of a Rydberg wave packet using a half- cycle pulse
}

\author{
J. Ahn \\ D.N. Hutchinson \\ Chitra Rangan \\ University of Windsor \\ P.H. Bucksbaum
}

Follow this and additional works at: https://scholar.uwindsor.ca/physicspub

Part of the Physics Commons

\section{Recommended Citation}

Ahn, J.; Hutchinson, D.N.; Rangan, Chitra; and Bucksbaum, P.H.. (2001). Quantum phase retrieval of a Rydberg wave packet using a half-cycle pulse. Physical Review Letters, 86 (7), 1179-1182.

https://scholar.uwindsor.ca/physicspub/10

This Article is brought to you for free and open access by the Department of Physics at Scholarship at UWindsor. It has been accepted for inclusion in Physics Publications by an authorized administrator of Scholarship at UWindsor. For more information, please contact scholarship@uwindsor.ca. 


\title{
Quantum Phase Retrieval of a Rydberg Wave Packet Using a Half-Cycle Pulse
}

\author{
J. Ahn, D. N. Hutchinson, C. Rangan, and P. H. Bucksbaum \\ Physics Department, University of Michigan, \\ Ann Arbor, Michigan 48109-1120 \\ (Received 15 September 2000)
}

\begin{abstract}
A terahertz half-cycle pulse was used to retrieve information stored as quantum phase in an $N$-state Rydberg atom data register. The register was prepared as a wave packet with one state phase reversed from the others (the "marked bit"). A half-cycle pulse then drove a significant portion of the electron probability into the flipped state via multimode interference.
\end{abstract}

DOI: 10.1103/PhysRevLett.86.1179

PACS numbers: 32.80.Rm, 03.67.-a, 42.30.Rx

An atomic Rydberg wave packet is an atom with one of its electrons in a coherent superposition of many highlying orbitals. Wave packets can be viewed as data registers for the information contained in the relative quantum phase of their constituent orbitals. One problem is that phase is difficult to detect. State selective detection on an ensemble of identically prepared registers usually reveals only the component amplitudes; the phases are hidden. Optical techniques have been employed to store and then efficiently retrieve phase information from atomic Rydberg wave packets [1], following a suggestion that binary information stored as phase in quantum registers could be searched more efficiently than the same classical information in classical data registers [2]. This Letter reports a different technique for extracting phase information, based on the interaction between the wave packet and a broadband coherent electric field pulse.

In the experiment reported in Ref. [1], a shaped optical pulse carried binary information into the Rydberg atom, by creating an electron wave packet with one or more states phase flipped (binary 1) with respect to the others (binary 0 ). A second optical pulse created another wave packet (the decoder) which holographically interfered with the register wave packet $[3,4]$. This interference converted the phase information to amplitudes by amplifying the flipped states and suppressing the unflipped states. This method of information storage is limited because it depends on mutual coherence between a low-lying launch state and the Rydberg states. Furthermore, the decoding pulse requires that most of the electron's probability amplitude resides in the launch state, and only a small portion goes into Rydberg states. Therefore, the scheme demonstrated in the above experiment cannot be scaled to a very large data register [5].

In the new data storage and retrieval experiment presented in this paper, the launch state is no longer part of the data register, and all of the probability resides in the Rydberg states of the atom. The decoding process of amplifying the flipped bits is now performed by a terahertz domain half-cycle pulse (HCP) [6]. This broadband coherent far-infrared radiation directly couples together Rydberg levels. Information stored in the phase domain is converted to amplitude information by coherent redistribution induced by the HCP.

HCP interactions with the Rydberg atoms have been studied previously, in HCP-ionization experiments and model calculations [7,8], HCP redistribution of energy eigenstates [9], and interactions with Rydberg wave packets $[10,11]$. In the present work, an HCP amplifies a phase-flipped orbital (the marked bit), suppressing the other unmarked states in the wave packet.

The HCP locates the marked bit because its activity depends on the wave packet's location. The interaction is strongest when the wave packet is localized at the ion core, and this situation corresponds to a coherent sum of orbitals with equal phase [10]. A Rydberg wave packet data register with one flipped bit is described by a superposition of such a localized wave packet, plus a phase-reversed orbital:

$$
\left|\Psi_{i}\right\rangle=\sum_{j}\left|n_{j} l m\right\rangle-2\left|n_{o} l m\right\rangle .
$$

A relatively weak HCP interaction will destroy the localized component easily, while the spatially delocalized single marked orbital $\left|n_{o} l m\right\rangle$ is amplified by a factor of approximately $2^{2}=4$. This is an example of the quantum diffusion and quantum amplification of information, discussed by Grover [12].

The database search experiment was performed in a beam of atomic Cs. The Rydberg data register was loaded using wave packet sculpting techniques that have been described previously [4]. Briefly, an optical pulse was sculpted with an acousto-optic Fourier filter [13] to excite a Rydberg data register with one marked bit in Cs $p_{z}$ states with $n=24-29$. State-selective field ionization (SSFI) [14] of the initial wave packet reveals only the amplitudes of the states, as shown in Fig. 1(a).

An HCP was generated inside the atomic beam apparatus by an ultrafast optical pulse illuminating a large aperture $\left(1.5 \times 3 \mathrm{~cm}^{2}\right)$ electrically biased GaAs wafer [6]. The far-infrared radiation was focused on the atomic beam by an off-axis parabolic mirror. Its peak field strength was calibrated by observing the threshold for ionization of Rydberg states [7]. The HCP delivered an impulse of 


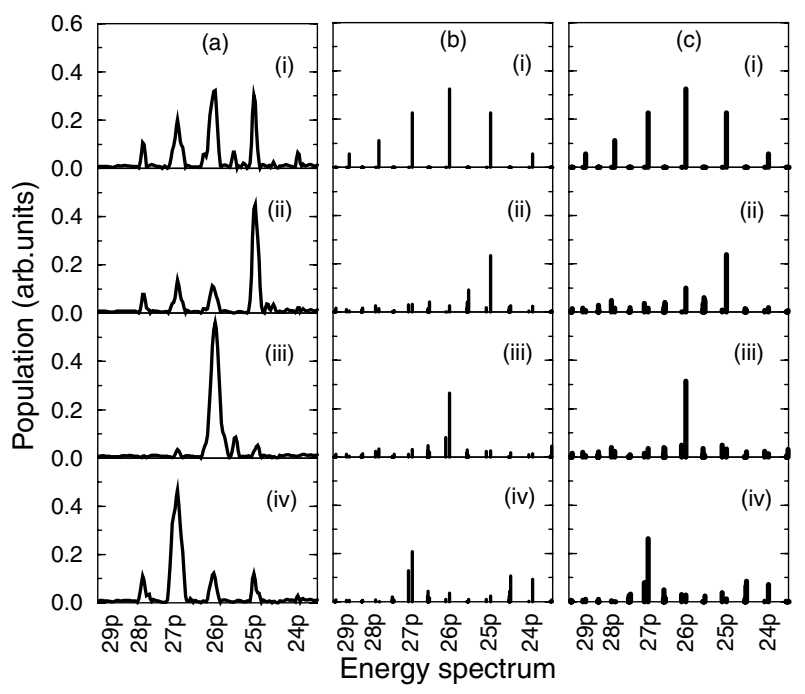

FIG. 1. Experimental data and model calculations of the stateselective field ionization spectra of the initial wave packet (i), and the final wave packets at $\tau=2.1 \mathrm{ps}$ (ii), $4.2 \mathrm{ps}$ (iii), and 4.7 ps (iv). (a) Experimental data. (b) Impulse model calculation. (c) Model calculation includes the direct integration of the time-dependent Schrödinger equation using the split-operator method. Both calculations assumed an initial Gaussian wave packet $\quad\left|\psi_{t=0}\right\rangle=0.5|24 p\rangle+|25 p\rangle+1.2|26 p\rangle+|27 p\rangle+$ $0.7|28 p\rangle+0.5|29 p\rangle$. The electron probability of the initial Gaussian wave packet was driven into the $25 p$ (ii), $26 p$ (iii), and $27 p$ (iv) states, respectively. The nearly degenerate $p$ and $d$ states and the $s$ and hydrogenlike states are not resolved in the experimental data.

approximately $Q_{0}=0.0043 \hbar / a_{0}$, which was enough to significantly mix $s, p$, and $d$ states [9]. It was polarized parallel to the Rydberg state.

The HCP interrogated the system at time $\tau$, converting the wave packet to a simpler structure in which only the marked state remains. Figure 1 shows SSFI spectra for three different data register configurations, in which a different state in the register was phase reversed as shown in Eq. (1): the flipped bit was $25 p, 26 p$, and $27 p$ in panels (ii), (iii), and (iv), respectively.

The quantum diffusion and marked state amplification can be calculated in the limit where the duration of the HCP is much less than the natural time scale for the phase evolution of the Rydberg wave packet, i.e., the Kepler orbit time, $t_{\mathrm{K}}=2 \pi / \Delta E=2 \pi\left(n-\delta_{l}\right)^{3}$, where $n$ is the principal quantum number and $\delta_{l}$ is the quantum defect for the $l$ th angular momentum states. For atomic cesium at

$$
E(t)=\left\{\begin{array}{cc}
0, & t<0 \\
29.56 E_{\text {peak }}\left[17.75(t / \tau)^{3} e^{-8.87 t / \tau}-0.412(t / \tau)^{5} e^{-4.73 t / \tau}\right], & t \geq 0
\end{array}\right\} .
$$

The field-free basis states were calculated using a grid-based pseudopotential method [15]. The time evolution was carried out by using a split-operator method in a restricted basis of essential states [16]. This symmetrizedproduct propagator is unitary and correct through the sec- $n=26, t_{\mathrm{K}} \approx 2 \mathrm{ps}$ is 5 times the full width half maximum (FWHM) of the HCP $\tau_{\mathrm{FWHM}} \approx 440 \mathrm{fs}$. In this impulsive limit, the HCP atomic interaction Hamiltonian (in atomic units with $e=m_{e}=\hbar=1$ )

$$
\mathcal{H}(t)=\vec{E}(t) \cdot \vec{r}=-\vec{Q} \delta(t) \cdot \vec{r},
$$

where $\vec{Q}=\int_{t-\epsilon}^{t+\epsilon} \vec{E}\left(t^{\prime}\right) d t^{\prime}$ is the total momentum transfer to the electron. The initial electron wave function $\left|\Psi_{i}\right\rangle$ acquires an extra phase along the direction of the impulse $(\hat{z})$

$$
\left|\Psi_{f}\right\rangle=e^{i \vec{Q} \cdot \vec{r}}\left|\Psi_{i}\right\rangle
$$

For an initial wave packet $\left|\Psi_{i}\right\rangle=\sum_{n} a_{n}|n l m\rangle$, the final state wave packet is

$$
\left|\Psi_{f}\right\rangle=\sum_{n^{\prime} l^{\prime} m^{\prime}, n} a_{n} f_{n l m}^{n^{\prime} l^{\prime} m^{\prime}}|n l m\rangle,
$$

where the transition matrix elements are given by

$$
f_{n l m}^{n^{\prime} l^{\prime} m^{\prime}}=\left\langle n^{\prime} l^{\prime} m^{\prime}\left|e^{i \vec{Q} \cdot \vec{r}}\right| n l m\right\rangle .
$$

For $l=1$ and $m=0$, and $\vec{Q}$ directed along $\hat{z}$, we find

$$
\begin{aligned}
f_{n p 0}^{n^{\prime} l^{\prime} m^{\prime}}(Q)= & \left\langle R_{n^{\prime} l^{\prime}}(r)\right| i^{l^{\prime}-1} \sqrt{\frac{3}{2 l^{\prime}+1}} \\
& \times\left[l^{\prime} j_{l^{\prime}-1}(Q r)-\left(l^{\prime}-1\right) j_{l^{\prime}+1}(Q r)\right] \\
& \times\left|R_{n p}(r)\right\rangle \delta_{m^{\prime}, 0} .
\end{aligned}
$$

The phase of each transition $f_{n l m}^{n^{\prime} l^{\prime} m^{\prime}}(Q)$ is determined by the parity change, $\Delta l=l^{\prime}-l$ and the magnitude of $Q$. For example, $p$ - $p$ transitions are always real and $p$-d transitions are imaginary, while the sign changes with $Q$.

The probability density in a final state $\left|n^{\prime} l^{\prime} m^{\prime}\right\rangle$ is given by

$$
P_{n^{\prime} l^{\prime} m^{\prime}}=\left|\sum_{n} a_{n} f_{n l m}^{n^{\prime} l^{\prime} m^{\prime}}(Q)\right|^{2}
$$

For atomic cesium levels around $n \approx 26$ excited by an impulse $Q=Q_{0}$, the self-transition amplitude $f_{n p 0}^{n p 0}$ is $\pi$ out of phase with transition amplitudes from other orbitals $f_{n p 0}^{n^{\prime} p 0}$. This creates constructive multimode interference for the marked states, while transitions to other states experience destructive interference. The results of a calculation in the impulse limit are shown in Fig. 1, and show qualitative agreement. Deviations from the impulse limit were studied with a second calculation, where the Schrödinger equation was integrated over a finite-duration model half-cycle pulse. We used an HCP model fitted to experimentally measured spectra [8]. The momentum transferred by the HCP, $Q$, is the area under the curve,

ond order in the time step. We used a basis of 187 states, that is, states with quantum numbers $n=21-31$ and $\ell<17(m=0)$ and a time step of $10 \mathrm{fs}$. The results are shown in Fig. 1. When a single initial state out of 

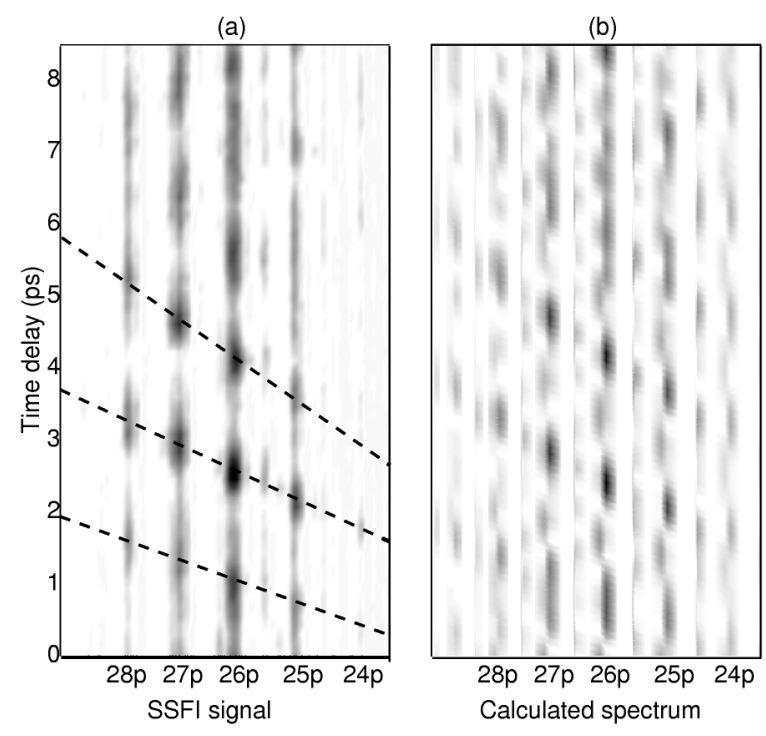

FIG. 2. Experiment (a) and theory (b) of the multimode interference pattern of Rydberg wave packets forced by the half-cycle pulse interaction. Guidelines follow the phase gradient maxima that appear around the half multiples of the Kepler orbit times $\tau=(k+1 / 2) t_{\mathrm{K}}(n)$ with $k=0,1$, and 2 .

the wave packet is phase flipped, we reproduce the result that this orbital survives the HCP with a significantly larger population than the rest, in good agreement with the experiment.

The full dynamics of the database are revealed by mapping the HCP redistribution as a function of the target time. Since the Rydberg levels in the data register are nondegenerate, different configurations of flipped bits occur at times other than $\tau$. This two-dimensional phase space interference pattern, or "quantum carpet" [17], is shown in Fig. 2, for data collected over several Kepler periods. The HCP consistently redistributed the wave packet into a single state when it consisted of only a single flipped orbital. The patterns of ridges in the quantum carpet show how the Kepler period changes with energy, and also display wave packet dispersion over 8 ps of the evolution. The lines are equivalent to classical ridges of a multimode interference pattern [17].

A quantitative measure of success in the database search problem is the reduction of the informational entropy of the system following the search algorithm [18]. Entropy $S$ in this simple case of a single marked state is defined as

$$
S=-\sum_{i=1}^{N} P_{i} \log P_{i},
$$

where $P_{i}$ is the probability that the marked bit resides in orbital $i$. The information system consists of the $N$-state data register. When the data register is loaded, the information is encoded as the phase of each orbital. The orbital amplitudes carry no information. Since SSFI measures only amplitudes, the information is completely hidden from view, and therefore the entropy of the system is maximum: $S_{\text {initial }}=-\log (1 / N)=\log N$.
TABLE I. Informational entropy calculations using Eq. (9) for experimental data $S_{\text {exp }}$, the impulse model calculation $S_{\text {imp }}$, and the full calculation with the time integration of the Schrödinger equation $S_{\text {full }}$. The initial entropy $S_{\text {initial }}=1.79$ for $N=6$.

\begin{tabular}{cccc}
\hline \hline Marked bit & $S_{\exp }$ & $S_{\text {imp }}$ & $S_{\text {full }}$ \\
\hline $25 p$ & 1.09 & 1.004 & 1.116 \\
$26 p$ & 0.56 & 1.032 & 1.081 \\
$27 p$ & 1.17 & 1.071 & 1.037 \\
\hline \hline
\end{tabular}

The interaction with the HCP converts the phase information into amplitudes, which can be read out using SSFI. The rules of quantum measurement ensure that only one state will be detected each time an atom is interrogated by SSFI. If the algorithm works perfectly, the marked orbital is revealed with unit efficiency, and the entropy drops to zero. Otherwise, unmarked orbitals may occasionally be detected by mistake. The entropy reduction can be calculated from the probability distribution, using Eq. (9).

The states that are not included in the data register can rob some of the probability during the HCP operation, and therefore contribute to the inefficiency of the algorithm. A lower bound on the amount of entropy contributed by leakage into the nonregister states can be measured by including a reservoir state in the calculation of the entropy. Table I shows the entropy reduction for each of the marked orbitals shown in Fig. 1. There is a significant entropy reduction, but the algorithm is far from perfect. Further progress could be made by shaping broadband terahertz radiation (which has been demonstrated using optical pulse shaping techniques [19]) to focus the state redistribution.

In conclusion, we have used a HCP to search for information stored as phase in a Rydberg wave packet. Phase information coded in a Rydberg quantum register was retrieved through the impulsive interaction of HCP. This interaction forced a constructive multimode interference only on a phase-flipped state. Direct integration of the timedependent Schrödinger equation as well as an impulsive model calculation showed good agreements with the experimental data. Beyond the problem of database searching, these results point toward the use of HCPs as general tools of analysis for unknown wave packets. This could be used in conjunction with optical techniques for wave packet tomography [4].

It is a pleasure to acknowledge useful discussions and help from T. C. Weinacht, J. L. White, P. R. Berman, K. J. Schafer, and R.R. Jones. This research was supported by the NSF Grant No. 9987916, and the Army Research Office. One of us (C. R.) gratefully acknowledges support from the Fellows and Visitors program of the Center for Ultrafast Optical Science at the University of Michigan.

[1] J. Ahn, T. C. Weinacht, and P. H. Bucksbaum, Science 287, 463 (2000). 
[2] L. K. Grover, Phys. Rev. Lett. 79, 325 (1997); 79, 4709 (1997).

[3] C. Leichtle et al., Phys. Rev. Lett. 80, 1418 (1998).

[4] T. C. Weinacht, J. Ahn, and P. H. Bucksbaum, Phys. Rev. Lett. 80, 5508 (1998).

[5] David A. Meyer et al., Science 289, 1431 (2000).

[6] B. I. Greene et al., Appl. Phys. Lett. 59, 893 (1991); D. You et al., J. Opt. Soc. Am. B 11, 486 (1994).

[7] R. R. Jones, D. You, and P. H. Bucksbaum, Phys. Rev. Lett. 70, 1236 (1993); C. O. Reinhold et al., J. Phys. B 26, L659 (1993).

[8] A. Bugacov et al., Phys. Rev. A 51, 1490 (1995).

[9] N.E. Tielking and R. R. Jones, Phys. Rev. A 52, 1371 (1995).

[10] C. Raman et al., Phys. Rev. Lett. 76, 2436 (1996).

[11] G. Alber and O. Zobay, Phys. Rev. A 59, R3174 (1999); M. B. Campbell, T. J. Bensky, and R.R. Jones, Phys.
Rev. A 58, 514 (1998); J. Bromage and C. R. Stroud, Jr., Phys. Rev. Lett. 83, 4963 (1999).

[12] L. K. Grover, Science 280, 228 (1998).

[13] M. A. Dugan, J.X. Tull, and W.S. Warren, J. Opt. Soc. Am. B 14, 2348 (1997).

[14] T. F. Gallagher et al., Phys. Rev. A 16, 1098 (1977).

[15] C. Rangan, K. J. Schafer, and A. R. P. Rau, Phys. Rev. A 61, 53410 (2000); J. N. Bardsley, in Case Studies in Atomic Physics, edited by E. W. McDaniel and M. R. C. McDowell (North-Holland, Amsterdam, 1974), Vol. 4, p. 302.

[16] M. D. Feit, J. Comput. Phys. 47, 412 (1982); M. R. Hermann and J. A. Fleck, Jr., Phys. Rev. A 38, 6000 (1988).

[17] A. E. Kaplan et al., Phys. Rev. A 61, 32101 (2000).

[18] C. E. Shannon, Bell Syst. Tech. J. 27, 379 (1948).

[19] C. Raman, M.F. DeCamp, and P.H. Bucksbaum, Opt. Express 1, 186 (1997). 